\title{
Energy-Aware Clustering for Multi-Cell Joint Transmission in LTE Networks
}

\author{
Efstathios Katranaras, Muhammad Ali Imran and Mehrdad Dianati \\ Centre for Communication Systems Research, CCSR \\ University of Surrey, UK \\ Email: e.katranaras@surrey.ac.uk
}

\begin{abstract}
This paper investigates the energy-aware clustering of cooperating base stations in the downlink of cellular networks. The focus of this work is on static clustering deployments for LTE systems when joint signal precoding is employed at multiple base stations. We demonstrate that properly planned clustering can provide the desired balance between network spectral and energy efficiency. To this end, we compare the overall energy consumption of various clustered cooperation layouts while considering different target performance metrics at user end. Our evaluations for various inter-site distance deployments in a practical macrocell scenario unveil the individual parameters controlling the energy effectiveness of a clustering strategy. In fact, it is shown that the choice of the optimum clustering layout depends on: 1) the specific service demands; 2) the deployment density of the network and; 3) on the ability of the base stations to jointly adjust their transmit power. Ultimately, we provide a general framework for choosing the most appropriate cooperation set of base stations in energy-aware networks.
\end{abstract}

\section{INTRODUCTION}

Inter-cell Interference (ICI) is a significant issue in cellular access networks. Particularly, in dense deployment scenarios, a User Equipment (UE) typically receives relatively strong signals from more than one base stations, or more generally termed Transmission Points (TPs). That has rendered Coordinated Multi Point (CoMP) a promising technique that improves fairness and the overall throughput of the system by managing ICI through coordination or cooperation between TPs [1]. In particular, the joint signal processing CoMP scheme, where transmit or receive information is exchanged between TPs, provides the potential of exploiting ICI [2]. In practical CoMP deployments, however, only a limited number of TPs should cooperate in order to contain the signalling and backhaul overhead required for joint processing. Such clustered implementation of CoMP is currently being considered by 3GPP for Long Term Evolution Advanced (LTE-A) networks [3].

On the other hand, Energy Consumption (EC) and efficiency of wireless access networks has recently become an active subject of interest for both environmental and economical reasons [4], [5]. This demands a careful re-assessment of new technologies like CoMP in order to investigate their implications in terms of both spectral and energy efficiency. This is particularly important since the characterisation of CoMP techniques' effect on network energy performance is not straightforward. Additional energy burden is introduced by CoMP schemes due to the need for: 1) extra channel state information and extra signal processing at the TPs and;
2) extra backhauling in order to obtain high speed, lowlatency, low-error connectivity between cooperating TPs [6]. At the same time, the resulting distributed Multiple-InputMultiple-Output (MIMO) system provides antenna diversity gains [7] which can translate into less transmit power needs in order to maintain a specific Quality of Service (QoS). Thus, understanding the tradeoff between energy and spectral efficiency will give the designers and operators an insight to strike a right balance for the performance of future networks.

To this end, this paper investigates the energy-aware clustering in CoMP-enabled cellular systems. We focus on static clustering, i.e. pre-decided and fixed over time, which can be straightforwardly implemented in current deployments. We carry out an analysis to explore if, when and which TPs in the downlink of a multi-cell system should in principle cooperate through signal joint processing to energy efficiently exploit the gains offered through ICI management. We construct a holistic framework to evaluate network performance in terms of both spectral and energy efficiency. To achieve that, we model and compare the overall energy consumption of various clustered cooperation layouts while considering two different target performance metrics at user end, corresponding to average per-cell throughput and rate fairness, respectively. In addition, we show that clustering and key system parameters such as QoS demand target, deployment density, and TPs transmit power can be jointly adjusted to provide enhanced energyaware performance in real-world cellular networks.

The rest of the paper is organised as follows. Section II describes the cooperation deployment scenario in order to formulate the CoMP clustering problem. Section III models the downlink channel under joint signal processing and provides an expression for the instantaneous UE Signal-toInterference-plus-Noise-Ratio (SINR). Section IV establishes the framework for evaluating the optimal clustering layout by introducing the different target performance metrics and the holistic model for energy consumption in cooperative systems. Finally, Section V provides simulation results evaluating practical macrocell cooperative scenarios along with insightful observations while Section VI concludes the paper.

\section{System Deployment \& Clustered Cooperation}

We consider a tri-sectored LTE cellular network of $N_{s}$ sites as described in [8]. Each site is controlled by a single eNodeB (eNB) and comprises 3 cells/sectors (i.e. the set $\mathcal{N}$ of all cells 


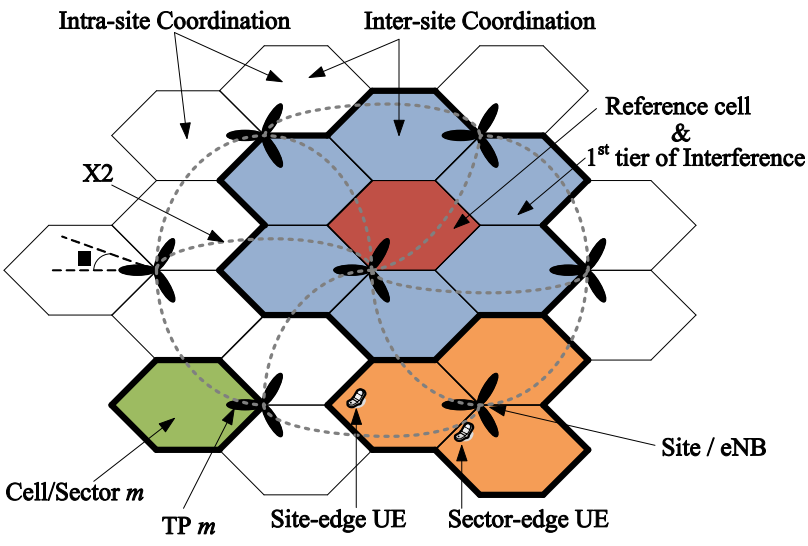

Fig. 1. Multi-cell system. Example with $N_{s}=7$.

in the system comprises $N=3 N_{s}$ cells), each served by a dedicated TP equipped with a single directional antenna (Fig. 1). Focusing in the downlink, we assume that $K$ single antenna UEs in the system are assigned to the same physical resources after scheduling, forming the set $\mathcal{K}$ of active UEs. We also assume the cooperating set $\mathcal{Q}$ of $Q$ TPs (with $Q \leq N$ ) that jointly transmit symbols to a subset $\mathcal{K}_{\mathcal{Q}} \subseteq \mathcal{K}$ of UEs.

In the tri-sectored cellular paradigm there are two distinguished fundamental scenarios for coordination between two TPs: a) Intra-site coordination between co-located TPs controlled by the same eNB and; b) Inter-site coordination between TPs controlled by neighbouring eNBs. Internal coordination can benefit from almost zero latency and infinite capacity information exchange, e.g. through short-distance wiring, and can therefore be achieved with minimal energy consumption [9]. In inter-site coordination, information has to be exchanged through the $\mathrm{X} 2$ interface connecting eNBs, as introduced by the LTE Radio Access Network [8]. Due to practical limitations, this interconnection between eNBs will be limited in capacity and latency, constraining the largescale implementation of such cooperation schemes. In this paper, focusing on CoMP energy aspects, we consider a star-like one-hop backhaul topology (see Fig. 1) where each eNB is interconnected only with its six immediate neighbours assuming that delay and capacity issues are satisfied; yet, we acknowledge and evaluate the energy consumption of the multiple backhaul links required for inter-site coordination.

Regarding the implementation of downlink CoMP, several schemes exist, each with varying degree of information exchange needs and way of distribution of this information. In this work, we consider the Joint Transmission (JT) scheme wherein signals from multiple TPs, forming a coordination cluster, can be jointly pre-processed to improve performance. In JT scheme, user data need to be available at all cluster TPs and therefore, it is the most demanding CoMP scheme in terms of backhaul bandwidth, and equivalently backhaul energy consumption, when a sizeable amount of information has to be exchanged between sites. Moreover, we focus on the case of coherent signal combining, achieved through the application of precoding filters at TPs to align the phases of

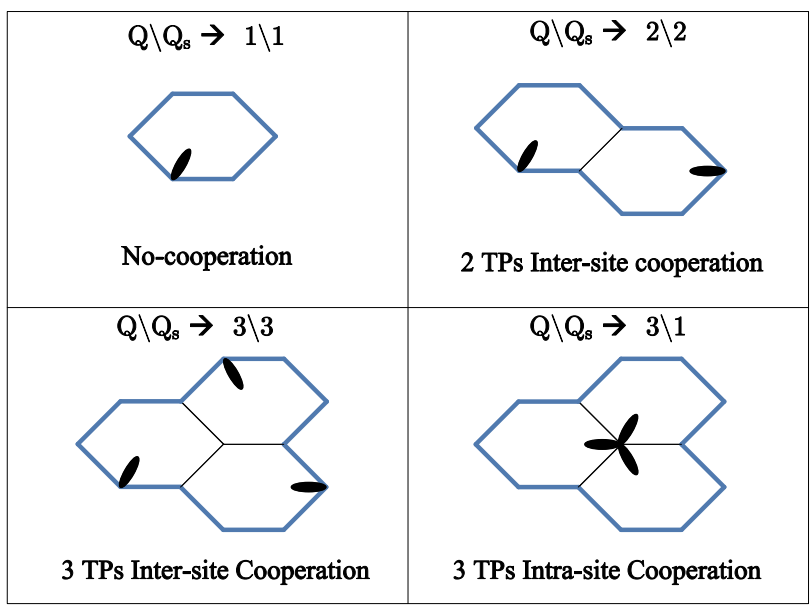

Fig. 2. Feasible cluster layouts for homogeneous cluster configuration.

signals transmitted from the multiple coordinating antennas. This allows the coordinated TPs to jointly transmit precoded data symbols to multiple CoMP-served UEs such that the desired signals overlap coherently and intra-cluster interference between these UEs is cancelled out. For this reason, full cluster knowledge of the Channel State Information at the Transmitter (CSIT) is required, i.e. between the TPs and UEs involved in the JT procedure of each cluster, which can be achieved through precise synchronisation in frequency and time between the involved coordinating entities, e.g. using a global positioning system [9].

Throughout this work we consider static clustering, as introduced in [10], where the cluster configuration stays unchanged over time. In that case, the cooperating sets of TPs are predecided based on system topology and propagation properties considering any potential UE locations in cells. We assume no coordination between clusters, i.e. all UEs in a cell and their serving TP participate in only one cluster at any time to avoid the high computation complexity introduced by such a clustering scheme. For overall system fairness, we further consider that clusters are formed homogeneously over the network. In that case, there are specific feasible cluster layouts that can be deployed throughout the entire network, i.e. a group of TPs can be considered as a feasible cluster layout only if the remaining TPs belonging to the same site can be a part of an identical cooperating group. Since we assume the JT as the enabling CoMP scheme, to keep the per cluster requirements in terms of required backhaul capacity and energy affordable, we consider a maximum cluster size of $Q_{\max }=3$, i.e. the TPs can cooperate in groups of two or three. According to the above, the feasible cluster layouts are defined as shown in Fig. 2. Each cluster layout is identified by a double index, i.e. $Q / Q_{s}$, denoting the number of TPs and eNBs, respectively, required for the coordination. Note that such small-scale cooperation should fit excellently to a macro cellular environment, where we can safely assume (due to high probability of Non-line-ofsight (NLOS) propagation) that a UE can "hear" its serving TP and TPs from a maximum of 1-tier surrounding cells as 


$$
y_{k}=\underbrace{\left(\mathbf{h}_{k}^{\mathcal{Q}}\right)^{H} \mathbf{w}_{k} x_{k}}_{\text {Desired signal from Q TPs } d_{k}}+\underbrace{\sum_{i \in\left\{\mathcal{K}_{\mathcal{Q}} / k\right\}}\left(\mathbf{h}_{k}^{\mathcal{Q}}\right)^{H} \mathbf{w}_{i} x_{i}}_{\text {Intra-cluster Interference } \zeta_{k}\left(\mathbf{w}_{\mathcal{Q}}\right)}+\underbrace{\sum_{j \in\left\{\mathcal{K} / \mathcal{K}_{\mathcal{Q}}\right\}}\left(\mathbf{h}_{k}^{\mathcal{N} / \mathcal{Q})^{H}} \mathbf{w}_{j} x_{j}\right.}_{\text {Inter-cluster Interference (ICLI) }}+\underbrace{n_{k}}_{\text {AWGN }} \cdot
$$

depicted in Fig. 1.

Finally, regarding the way the TPs coordinate their transmissions, we consider a centralised scheme [11]; a controlling/scheduling entity, located at a Master-eNB (M-eNB) assigned for a specific cluster, calculates the scheduled data bits to be transmitted to the JT-served UEs. After the Orthogonal Frequency-Division Multiple Access (OFDMA) processing (including raw data encoding, modulation, mapping and Inverse Fast Fourier Transform (IFFT)), each M-eNB distributes frequency domain IQ samples along with appropriate scheduling and precoding information to the coordinating TPs. Colocated TPs are assumed to exchange information through the backplane of their eNB. Note that centralised coordination in that sense requires each eNB to have the respective capabilities so as to perform as a M-eNB if needed. In the following we model the downlink JT-enabled channel to obtain an in depth view of the respective communication system design.

\section{Channel Model \& UE SINR}

Since OFDMA is employed as the multiple access technique, it is sufficient to observe the baseband transmission on a single subcarrier. In that case, the resulting channel can be seen as a linear time-invariant and flat fading channel. Focusing on a scheduled UE $k \in \mathcal{K}_{\mathcal{Q}}$, the jointly transmitted symbol intended to this specific UE is denoted by $x_{k}$, with transmit power $\mathbb{E}\left\{x_{k} x_{k}^{*}\right\}=P_{k}$. Assuming CSIT, an additional precoding operation can be performed. Considering linear precoding at the transmitter side, the precoded symbol for the $k^{\text {th }}$ UE is given as $\mathbf{s}_{k}=\mathbf{w}_{k} x_{k}$, with $\mathbf{w}_{k} \in \mathbb{C}^{Q \times 1}$ denoting the precoding vector. Thus, the received signal at UE $k$ is given in (1), where $n_{k}$ is the zero-mean complex Gaussian noise and $\mathbf{h}_{i}^{\mathcal{J}}$ denotes the complex channel coefficient between TPs in set $\mathcal{J}$ and UE $i$. The channel coefficient between TP $j$ and UE $i$, is modeled as $h_{i}^{j}=g_{i}^{j} \lambda_{i}^{j}$, where $g_{i}^{j} \in \mathbb{R}$ and $\lambda_{i}^{j} \in \mathbb{C}$ stand for the distance dependent path loss and fast fading coefficients, respectively, of the specified channel. Note that in the following we are assuming Rayleigh fading, i.e. the fast fading coefficients are independent and identically distributed (i.i.d) $\sim \mathcal{N}(0,1)$.

The overall received signal after precoding and transmission in (1) can be expressed in a more condensed form as:

$$
y_{k}=d_{k}\left(\mathbf{W}_{\mathcal{Q}}\right)+\zeta_{k}\left(\mathbf{W}_{\mathcal{Q}}\right)+z_{k},
$$

with $d_{k}$ and $\zeta_{k}$ being functions of the overall precoding matrix $\mathbf{W}_{\mathcal{Q}} \in \mathbb{C}^{Q \times\left|\mathcal{K}_{\mathcal{Q}}\right|}$ that is known at the M-eNB of the cluster of interest and $z_{k}$ denoting the coloured noise-plusICLI part realised at the receiver. According to (1) and (2), the achievable instantaneous SINR estimated at UE $k$ is:

$$
\begin{aligned}
\operatorname{SINR}_{k} & =\frac{\left\|\left(\mathbf{h}_{k}^{\mathcal{Q}}\right)^{H} \mathbf{w}_{k} x_{k}\right\|^{2}}{\left\|\sum_{i \in\left\{\mathcal{K}_{\mathcal{Q}} / k\right\}}\left(\mathbf{h}_{k}^{\mathcal{Q}}\right)^{H} \mathbf{w}_{i} x_{i}\right\|^{2}+\left\|z_{k}\right\|^{2}} \\
& =\frac{\left\|d_{k}\left(\mathbf{W}_{\mathcal{Q}}\right)\right\|^{2}}{\left\|\zeta_{k}\left(\mathbf{W}_{\mathcal{Q}}\right)\right\|^{2}+\left\|z_{k}\right\|^{2}} .
\end{aligned}
$$

We consider that an idealistic preprocessing technique takes advantage of the perfect CSIT and calculates appropriately the $\mathbf{W}_{\mathcal{Q}}$ matrices for each cluster so as the ICLI is removed completely while the maximum power diversity gain is achieved for each UE, i.e. coherent combining of jointly transmitted symbol and equal transmit symbol power among all scheduled UEs is achieved. Assuming all TPs transmit with equal total power, $P_{\mathrm{Tx}}$, the idealistic instantaneous SINR at UE $k$ is:

$$
\widehat{\operatorname{SINR}}_{k}=\frac{P_{\mathrm{Tx}} \sum_{q \in \mathcal{Q}}\left\|h_{k}^{q}\right\|^{2}}{P_{\mathrm{Tx}} \sum_{m \notin \mathcal{Q}}\left\|h_{k}^{m}\right\|^{2}+N_{0} B},
$$

where $N_{0}$ is the noise spectral density and $B$ the system bandwidth.

\section{QOS \& ENERGY CONSUMPTION}

In general, we target to find the most efficient cluster layout according to the design objective of minimum overall system energy consumption for the design criteria of given QoS requirements. Regarding the system design criteria, we consider in this work two distinct QoS-related metrics: 1) Mean percell SINR of all potential UE locations under a particular cluster layout; 2) Outage cell SINR, i.e. percentage of potential UE locations that do not achieve a certain minimum SINR value. The former metric is related to the average per-cell throughput while the latter relates to cell-edge throughput and rate fairness, which are of high interest for network operators.

On the other hand, the overall CoMP-enabled network energy consumption will be directly related to the power usage of all network elements over a time period. A holistic power model for any cooperation cluster set $\mathcal{Q}$ can be given by:

$$
P_{\mathcal{Q}}=Q\left(P_{\mathrm{TP}}+P_{\mathrm{sp}}\right)+\left|\mathcal{K}_{\mathcal{Q}}\right| P_{\mathrm{UE}}+P_{\mathrm{bh}},
$$

where $P_{\mathrm{TP}}, P_{\mathrm{sp}}, P_{\mathrm{UE}}, P_{\mathrm{bh}}$ stand for the TPs power usage related with the radiated power from antennas, the per-TP signal processing power, the cluster power needs for backhaul, and the average power usage at the active UEs served by the cluster TPs, respectively. For a complete evaluation on the energy consumption of the network we need to adopt mathematically tractable power models for each element in (5). Thus, the individual element power models are discussed in the following. 


\section{A. TP Transmission Power}

A linear approximation base station power model can be used according to [12] as:

$$
P_{\mathrm{TP}}=P_{0}+\Delta_{p} P_{\mathrm{Tx}},
$$

where $0 \leq P_{\mathrm{Tx}} \leq P_{\mathrm{Tx}-\max }$ denotes the RF per-antenna output power, constrained by a maximum $P_{\text {Tx-max }}$ practical value, $P_{0}$ represents the circuit power consumption at zero RF output power and $\Delta_{p}$ is the slope of the load dependent power consumption.

\section{B. Signal Processing Power}

Baseband digital signal processing complexity is increased in CoMP-enabled systems due to extra channel estimation and extra MIMO processing needs. Extending the model presented in [6], to capture how the processing power usage corresponding to each part is increased based on the extend of cooperation, the signal processing power needs can be calculated by:

$$
P_{\mathrm{sp}}=p_{\mathrm{sp}}\left(1-\alpha_{\mathrm{csi}}-\alpha_{\mathrm{mimo}}+\alpha_{\mathrm{csi}} Q_{\mathrm{csi}}+\alpha_{\mathrm{mimo}} Q_{\mathrm{mimo}}^{2}\right),
$$

where $p_{\mathrm{sp}}$ stands for a base value parameter, $Q_{\mathrm{csi}} \equiv Q_{s}$ denotes the number of TPs taking part in the channel estimation process, $Q_{\text {mimo }} \equiv Q$ stands for the number of TPs cooperating in MIMO fashion to enable JT and $\alpha_{\mathrm{csi}}, \alpha_{\text {mimo }}$ are scaling factors.

\section{Backhaul Power}

For inter-site coordination, we consider multiple wireless microwave links, of capacity $c_{\text {link }}$ each, used for the eNBs interconnection in a centralised manner. The required power in that case can be modelled, by considering a linear model with a maximum power dissipation $P_{\text {link }}$ for each link [6]:

$$
P_{\mathrm{bh}}=\left(Q_{s}-1\right) \frac{c_{\mathrm{bh}}}{c_{\text {link }}} P_{\text {link }},
$$

where $c_{\mathrm{bh}}$ represents the per-link backhaul load requirements of each layout. For JT, there are four types of exchanged information through the backhaul; namely, user data, CSI, scheduling information (including exchange of precoding vectors) and signalling information. However, the amount of user data will dominate over the other types of information to be exchanged in that scheme [9]. Considering a quantise-andforward method with $q_{\mathrm{I}}$ and $q_{\mathrm{Q}}$ bits per I and Q component, respectively, and that transmit symbols at all $n_{\mathrm{sc}}$ subcarriers of spacing $B_{\mathrm{sc}}$ are forwarded, the required per-link bit-per-second backhaul load due to user data exchange can be estimated by:

$$
c_{\mathrm{bh}}=n_{\mathrm{sc}} B_{\mathrm{sc}}\left(q_{\mathrm{I}}+q_{\mathrm{Q}}\right) \text {. }
$$

\section{UE Power}

A tractable general power consumption model for UEs can be given by [13] as:

$$
P_{\mathrm{UE}}=P_{c}+\frac{P_{\mathrm{tx}}\left(R_{\mathrm{csi}}\right)}{\epsilon}
$$

where $P_{c}$ is the circuit power representing the average energy consumption of UE electronics, $0 \leq P_{\mathrm{tx}} \leq P_{\mathrm{tx}-\max }$ stands for

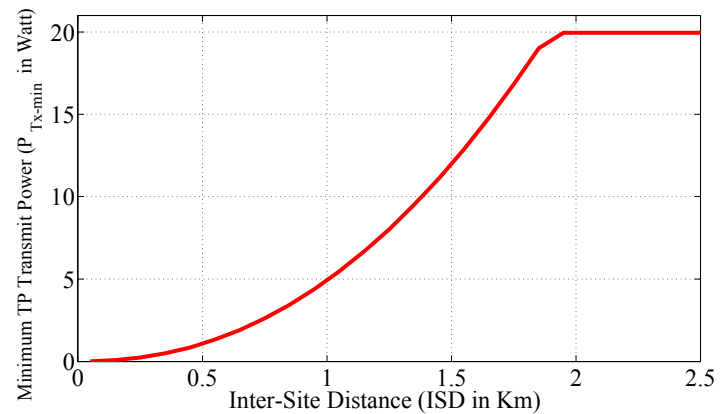

Fig. 3. Minimum required TPs transmit power as a function of ISD.

TABLE I

LTE-BAsed Scenario - System Model Parameters

\begin{tabular}{c|c|c}
\hline Parameter & Symbol & Values \& Ranges \\
\hline Frequency Carrier & $f_{c}$ & $2.4 \mathrm{GHz}$ \\
Channel Bandwidth & $B$ & $10 \mathrm{MHz}$ \\
No of Subcarriers & $n_{\mathrm{Sc}}$ & 600 \\
Subcarrier Spacing & $B_{\mathrm{sc}}$ & $15 \mathrm{kHz}$ \\
\hline Noise Figure at UE & $\mathrm{NF}_{\mathrm{UE}}$ & $7 \mathrm{~dB}$ \\
UE sensitivity & $\mathrm{UE}_{\mathrm{thr}}$ & $-120 \mathrm{dBm}$ per subcarrier \\
\hline UE antenna gain & $G_{\mathrm{UE}}$ & $-1 \mathrm{dBm}$ \\
TP antenna gain & $G_{\mathrm{TP}}$ & $\left(12-\min \left(\left(12 \frac{\theta}{70^{\circ}}\right)^{2}, 25\right) \mathrm{dBi}\right.$ \\
\hline TP antenna cable loss & $L_{\mathrm{cable}}$ & $20 \mathrm{~dB}$ \\
Outdoor-Indoor loss & $L_{\text {out-in }}$ & $8.8 \mathrm{~dB}$ \\
Shadow margin & $L_{\mathrm{sh}}$ & 3 \\
\hline Cell-edge SINR target & $\mathrm{SINR}_{\mathrm{edge}}$ & 8,8 \\
Outage SINR target & $\mathrm{SINR}_{\mathrm{out}}$ & \\
\hline QI quantization bits & $q_{Q}, q_{I}$ & \\
\hline
\end{tabular}

the UE transmit power as a function of the rate $R_{\mathrm{csi}}$ required for the CSI feedback operation to the TPs and $\epsilon \in(0,1]$ denotes the efficiency of the power amplifier at UEs.

\section{Simulation Results \& Discussion}

This section evaluates the performance of different cluster layouts in the context of a real-world cellular network scenario. To this end, a LTE-based system model and propagation parameters suggested by 3GPP in [3] are chosen as an example for establishing the relation of the various system modelling parameters with practical ones. The path loss coefficients are fitted to the respective "Urban Macro - LOS" empirical scenario. To this end, Table I summarises the system parameters considered for the LTE downlink. For the evaluation, we performed hybrid event-driven/Monte-Carlo simulations. We considered a large enough (i.e. 1000) number of potential UE locations in each cell. The averaged numerical results on each potential UE SINR were obtained by generating multiple (i.e. 100) random system instances to construct the system channel matrices at each instance for a specific ISD deployment and cluster layout.

In the following evaluations, a minimum TP transmit power, $P_{\text {Tx-min }}$, is considered as a function of ISD, so as any celledge UE can achieve a minimum SINR target under no cooperation. Fig. 3 illustrates the $P_{\text {Tx-min }}$ considered for each ISD deployment when applying the respective LTE downlink budget analysis for a cell-edge target SINR of $1(=0 \mathrm{~dB})$. 


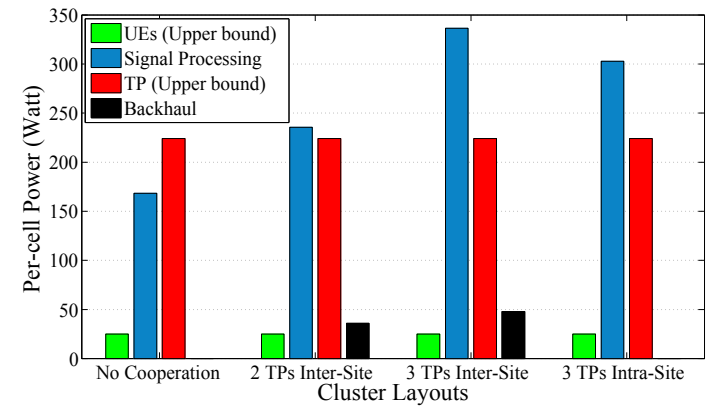

Fig. 4. Power usage of the various network elements for different cluster layouts.

TABLE II

Practical Power-Related Parameters

\begin{tabular}{c|c}
\hline Parameter & Value \\
\hline$P_{0}$ & 130 Watt \\
\hline$\Delta_{p}$ & 4.7 \\
\hline$P_{\text {Tx-max }}$ & $20 \mathrm{Watt}$ \\
\hline$c_{\text {link }}$ & $100 \mathrm{Mbps}$ \\
\hline$P_{\text {link }}$ & $50 \mathrm{Watt}$ \\
\hline$p_{\mathrm{sp}}$ & $168.2 \mathrm{Watt}$ \\
\hline$\alpha_{\mathrm{csi}}$ & 0.1 \\
\hline$\alpha_{\operatorname{mimo}}$ & 0.1 \\
\hline$P_{c}$ & $100 \mathrm{~mW}$ \\
\hline$P_{\mathrm{tx}-\max }$ & $200 \mathrm{~mW}$ \\
\hline \multicolumn{2}{|c}{}
\end{tabular}

Furthermore, Table II summarizes the exemplary values of all power-associated parameters considered for the LTE downlink budget of the macrocell scenario. To this end, Fig. 4 illustrates the per-cell power usage from each system element under the various cluster layouts considered. It becomes apparent that signal processing becomes the dominant element of power consumption as cluster size increases and, therefore, it is a decisive factor on the affordable cooperation cluster size for energy efficiency demanding cellular systems. Note that the illustrated value for TP transmission stand for an upper bound and may be significantly lower if the system QoS needs can be achieved with lower TPs' transmitting power. Note also that the UEs power value stands for an overestimation by considering a relative large number of UEs, i.e. 50, to be served per cell and a relatively low power amplifier efficiency $\epsilon=0.5$ for every UE. Even in that case, UEs energy consumption is rather insignificantly compared to the other system elements and for that reason it is omitted in the following overall network performance evaluations.

Fig. 5 and 6 depict the mean and outage cell SINR achieved, respectively, versus the energy consumed at each cluster layout for different ISD deployments $(0.5,1.5,3 \mathrm{Km})$. We vary TPs transmit power from the minimum $P_{\mathrm{Tx}-\mathrm{min}}$ (ISD) value to $P_{\text {Tx-max }}$. Important insights are obtained for the optimum: 1) TPs transmit power strategy and; 2) cluster layouts.

\section{A. Optimum Transmit Power Strategy}

Generally, for the ICI-limited dense deployments (e.g. ISD $=500 \mathrm{~m}$ ), it is optimal for TPs to transmit with low power close to $P_{\text {Tx-min }}$ (ISD). In that case, both energy consump-

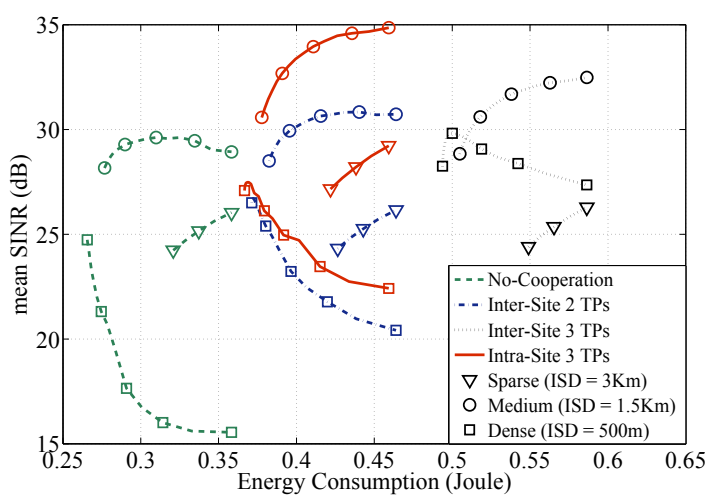

Fig. 5. Mean cell SINR versus cluster energy consumption for various cluster layouts, TPs transmit powers and ISDs.

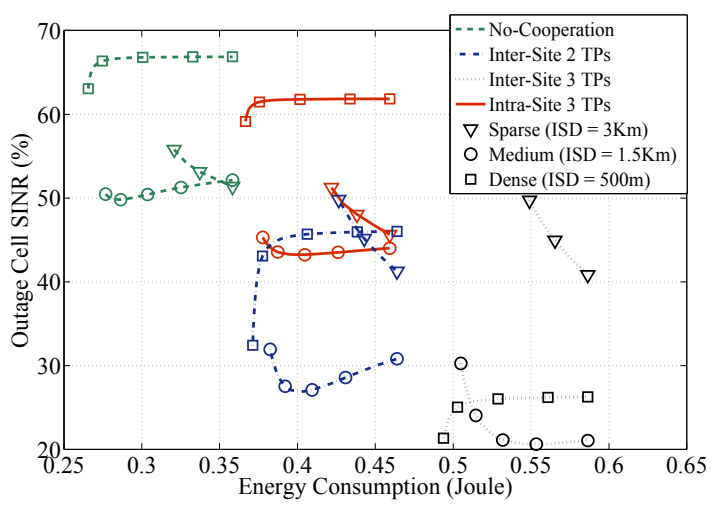

Fig. 6. Outage cell SINR (i.e. percentage of UEs do not achieving the SINR target) versus cluster energy consumption for various cluster layouts, TPs transmit powers and ISDs.

tion and interference are kept at minimum while maximum SINR performance is achieved. Contrarily, for the noiselimited sparse deployments (e.g. ISD $=3 \mathrm{Km}$ ), TPs have to transmit with high power close to $P_{\text {Tx-max }}$ to improve SINR performance while satisfying the cell-edge SINR target. In medium density deployments (e.g. ISD $=1.5 \mathrm{Km}$ ), noise and interference effects are of the same magnitude and TPs transmit power can be adjusted to obtain a satisfactory balance on SINR-EC performance. In this density region, an optimum TPs power, $P_{\mathrm{Tx}-\mathrm{opt}}$, exists; if TPs transmit with larger power than $P_{\text {Tx-opt }}$, cluster EC will increase and at the same time QoS will degrade leading to suboptimal overall system performance.

\section{B. Optimum Cluster Layouts}

The choice of the most energy efficient cluster layout depends both on the QoS requirements (i.e. mean- or outagecell SINR) and the system density deployment. For sparse systems, there is no significant improvement on outage cell SINR with any cluster layout (Fig. 6). That is explained from the fact that the effects of cooperation become rather insignificant to UEs at the borders of the site (site-edge UEs, see Fig. 1) in that case. However, if the system targets to optimise mean per-cell SINR, a rather significant gain can be 


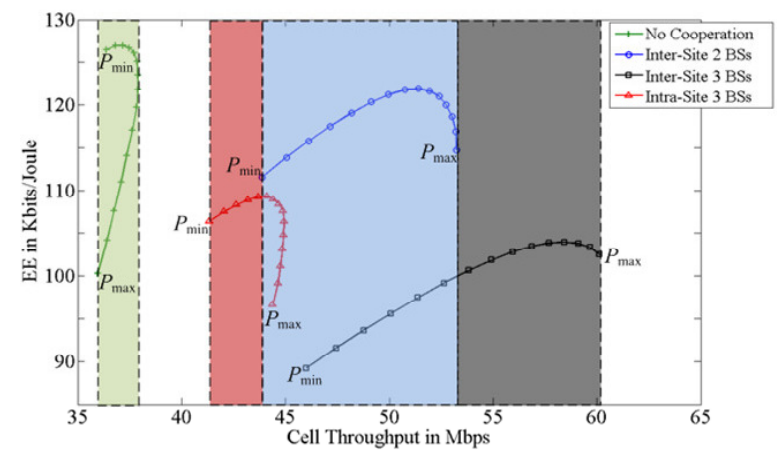

Fig. 7. Average network energy efficiency versus mean throughput for different cluster layouts. ISD $=2 \mathrm{Km}$. Each of the four shaded areas corresponds to the different optimal layout at the respective system throughput range demand.

achieved through intra-site coordination (Fig. 5); in that case, UEs at the sector borders (sector-edge UEs) can still benefit from CoMP. On the other hand, for dense systems, where the effect of inter-site interference will become more severe for site-edge UEs, coordination among TPs in different sites proves to be more effective. Especially for optimising outage cell SINR, inter-site coordination of TPs seems to pose as the only feasible solution.

\section{Energy Effi iency (EE) - Throughput Tradeoff}

Medium density systems appear to achieve, in general, the best SINR performance since an optimum balance between the useful cooperation and the harmful ICLI effects can be achieved in that case. In Fig. 7, we have translated the SINREC analysis into Joule-per-bit EE versus cell throughput results by considering that the Shannon capacity is achieved per link, i.e. $C_{k}=\log _{2}\left(1+\operatorname{SINR}_{k}\right)$. The performance of the different cooperation schemes for variable TPs transmit power values is illustrated. The results reveal more clearly that although a no-cooperation scheme can be more energy efficient when TPs transmit with appropriate power, it cannot reach the high rates obtained by clustered cooperation. In that regard, the differently shaded areas in the figure denote the optimal cluster layout that should be applied to achieve specific system throughput targets.

\section{CONCLUSION}

In this work, we have identified if, when and which cells in the downlink of LTE cellular networks should cooperate to efficiently exploit the gains offered by CoMP techniques. We considered static clustered multi-cell joint processing, variable cell density and a realistic holistic system power consumption model demonstrating that properly planned clustering can provide the desired balance between network spectral and energy efficiency. The study is conducted by comparing the overall performance of various cluster layouts. Our analysis and evaluation results show that the decision on the most energy efficient cluster layout depends both on the QoS-based requirements and the deployment density. Regarding energyaware average throughput optimisation, a significant gain can be achieved via intra-site coordination which favours sectoredge UEs. On the other hand, for optimising rate fairness, inter-site coordination favouring site-edge UEs is preferable. Inter-site coordination proves to be more effective especially for dense deployments, where the effect of inter-site interference becomes severe for site-edge UEs. We furthermore showed that system EE can be improved when TPs transmit with low power in the ICI-limited dense deployments. In sparser deployments, where noise and interference effects are of the same magnitude, TPs transmit power can be adjusted at higher level to optimise EE or obtain a satisfactory throughputEC tradeoff performance. We finally introduced a general framework to show how the most appropriate energy-aware static clustered cooperation can be determined and employed to optimise system energy efficiency when specific network capacity targets need to be reached.

\section{ACKNOWLEDGMENT}

This work has been done within a joint project, supported by Huawei Tech. Co., Ltd, China.

\section{REFERENCES}

[1] O. Somekh, O. Simeone, Y. Bar-Ness, A. Haimovich, U. Spagnolini, and S. Shamai, Distributed Antenna Systems: Open Architecture for Future Wireless Communications. Auerbach Publications, CRC Press, 2007, ch. An information theoretic view of distributed antenna processing in cellular systems.

[2] M. K. Karakayali, G. J. Foschini, and R. A. Valenzuela, "Network coordination for spectrally efficient communications in cellular systems," IEEE Wireless Communications Magazine, vol. 13, no. 4, pp. 56-61, 2006.

[3] 3GPP TR 36.814 v1.5.1 (2009-12), "Further advancements for E-UTRA, physical layer aspects," Tech. Rep., 2009.

[4] G. P. Fettweis and E. Zimmermann, "ICT Energy Consumption - Trends and Challenges," in 11th International Symposium on Wireless Personal Multimedia Communications (WPMC'08), Lapland, Finland, September 2008.

[5] G. Auer, I. Godor, L. Hevizi, M. Imran, J. Malmodin, P. Fazekas, G. Biczok, H. Holtkamp, D. Zeller, O. Blume, and R. Tafazolli, "Enablers for energy efficient wireless networks," in Proc. IEEE 72nd Vehicular Technology Conference Fall (VTC 2010-Fall), Sept. 2010, pp. $1-5$.

[6] A. Fehske, P. Marsch, and G. Fettweis, "Bit per joule efficiency of cooperating base stations in cellular networks," in IEEE GLOBECOM Workshops (GC'10 Wkshps), Dec. 2010, pp. 1406 -1411.

[7] H. Dai and H. Poor, "Asymptotic spectral efficiency of multicell MIMO systems with frequency-flat fading," IEEE Trans. Signal Processing, vol. 51, no. 11 , pp. 2976 - 2988, Nov. 2003.

[8] 3GPP TS 36.300 V11.1.0 (2012-03), "Evolved Universal Terrestrial Radio Access (E-UTRA) and Evolved Universal Terrestrial Radio Access Network (E-UTRAN)," Tech. Rep., 2012.

[9] P. Marsch and G. P. Fettweis, Eds., Coordinated Multi-Point in Mobile Communications - From Theory to Practice. Cambridge University Press, 2011.

[10] P. Marsch and G. Fettweis, "Static Clustering for Cooperative MultiPoint (CoMP) in Mobile Communications," in IEEE International Conference on Communications (ICC), June 2011, pp. $1-6$.

[11] S. Brueck, L. Zhao, J. Giese, and M. Amin, "Centralized scheduling for joint transmission coordinated multi-point in LTE-Advanced," in International ITG Workshop on Smart Antennas (WSA'10), Feb. 2010, pp. $177-184$.

[12] M.A. Imran and E. Katranaras (Eds.), "Energy Efficiency Analysis of the Reference Systems, Areas of Improvements and Target Breakdown," public deliverable D2.3 of Project EARTH, Tech. Rep., Jan. 2011

[13] G. Miao, N. Himayat, and G. Y. Li, "Energy-efficient link adaptation in frequency-selective channels," IEEE Transactions on Communications, vol. 58 , no. 2 , pp. $545-554,2010$. 